\title{
Assessment of visual and auditory evoked potentials in multiple sclerosis patients with and without fatigue
}

\author{
Anna Pokryszko-Dragan • Malgorzata Bilinska • \\ Ewa Gruszka • Elżbieta Kusinska • Ryszard Podemski
}

Received: 6 January 2014 / Accepted: 12 September 2014/ Published online: 21 September 2014

(C) The Author(s) 2014. This article is published with open access at Springerlink.com

\begin{abstract}
The aim of the study was to evaluate visual and brainstem auditory evoked potentials (VEP, BAEP) in multiple sclerosis (MS) patients with regards to fatigue and disease-related variables. The study comprised $86 \mathrm{MS}$ patients and 40 controls. Fatigue was assessed using the Fatigue Severity Scale (FSS/FSS-5) and the Modified Fatigue Impact Scale (MFIS). Latencies and amplitudes of the P100 component of VEP and the I-V components of BAEP were analyzed. The results of EP were compared between non-fatigued, moderately and severely fatigued MS patients and controls. P100 latency was increased and amplitude decreased in moderately and severely fatigued MS subjects. The latency of the V component of BAEP and interlatencies I-III-V were increased in severely fatigued patients. The amplitude of the $\mathrm{V}$ component was lowered in fatigued patients. VEP and BAEP abnormalities were usually one-sided. Interocular P100 latency difference tended to correlate with FSS/FSS-5. The parameters of VEP and BAEP correlated with functional system scores but not with MS duration, overall degree of disability or its progression over time. Significant, usually asymmetrical VEP and BAEP abnormalities were found in fatigued MS patients, with no relationships to disease-related variables. EP may be considered an electrophysiological marker of fatigue in MS patients.
\end{abstract}

Keywords Multiple sclerosis · Fatigue .

Evoked potentials

\footnotetext{
A. Pokryszko-Dragan $(\varangle) \cdot$ M. Bilinska $\cdot$ E. Gruszka .

E. Kusinska $\cdot$ R. Podemski

Department of Neurology, Medical University of Wroclaw,

Borowska 213, 50-556 Wrocław, Poland

e-mail: annapd@interia.pl
}

\section{Introduction}

Fatigue is commonly reported by patients with multiple sclerosis and has a profound impact upon their daily activities and quality of life. Although often discussed in recent years, the origin of fatigue remains unclear. Evaluation of fatigue also brings about difficulties because of its lack of the objective biomarkers $[1,2]$. According to some theories, the background of fatigue is associated with disturbed bioelectrical neuronal activity due to demyelination and axonal loss [3, 4]. Studies with the use of motor evoked potentials and electroencephalography event-related desynchronization have indeed shown decreased neuronal excitability and frequency-dependent conduction block in fatigued MS patients [5-8]. Visual and auditory evoked potentials are regarded as useful tools for recognizing and monitoring damage to central nervous system ascending pathways in the course of MS. However, these methods have not been used so far in studies on MS fatigue, investigating its origin and methods of its evaluation.

The aim of our study was to assess visual and brainstem auditory evoked potentials (BAEP) in MS patients with regard to the presence and severity of fatigue, considering also the impact of other disease-related variables.

\section{Materials and methods}

The study comprised 86 patients with MS (24 men and 62 women, aged 19-60 years, mean 39.55) who were under the care of the outpatient MS clinic, Department of Neurology, Medical University of Wroclaw. All the patients met the McDonald's criteria [9] of clinically definite MS. None of the patients had concomitant diseases known to cause fatigue or to affect parameters of visual and auditory evoked 
potentials in their history. 62 patients had never been treated with disease-modifying agents. 24 subjects had undergone treatment with interferon beta or glatiramer acetate for 1-3 years, but treatment had ceased at least 6 months prior to their inclusion in this study (in 15 patients because of their transition into secondary progressive phase of MS; nine subjects resigned from the treatment due to its side effects or for personal reasons). None of the patients were being treated with chronic immune-suppression. A washout period of at least 4 weeks was maintained between inclusion in the study and tapering treatment with corticosteroids due to the most recent MS relapse.

The control group consisted of 40 healthy volunteers, who were matched for age and gender to the MS patients (12 men, 28 women, aged 23-60 years, mean 38.8).

All the subjects gave their informed consent to participate in the study and the project was approved by the Bioethical Committee at the Medical University of Wroclaw.

The patients underwent a neurological examination and their disability was assessed using the Expanded Disability Status Scale (EDSS) [10], with visual and brainstem functional systems (FS) scores separated for further analysis. On the basis of medical records, the duration of the disease was defined and the index of disability progression [Multiple Sclerosis Severity Scale (MSSS)] calculated [11]; the history of optic neuritis or loss of hearing was also determined.

\section{Assessment of fatigue}

The level of fatigue in MS patients was evaluated using self-assessment questionnaires based on the Fatigue Severity Scale (FSS) [12] and the Modified Fatigue Impact Scale (MFIS) [13], with the results of FSS re-evaluated using the Rasch analysis applied by Mills et al. [14] (FSS5). On the basis of FSS/FSS-5 results, the patients were divided into three subgroups: without fatigue (subgroup I, FSS/FSS-5 <3.5), with moderate (subgroup II, FSS/FSS$5=3.5-5.5$ ) or severe fatigue (subgroup III, FSS/FSS-5 $>5.5$ ).

\section{Evoked potentials}

Visual evoked potentials (VEP) were performed by using a black and white checker board pattern on a screen, with a checker size of $36 \mathrm{~cm}^{2}$ and the frequency of pattern reversing being $1.9 \mathrm{~Hz}$. The subjects were sitting in the distance of $1 \mathrm{~m}$ from the screen, with the angle of vision $2^{\circ} 9^{\prime}$. The stimuli were presented uni-ocularly. An active recording electrode was attached to the scalp on the midline at the occipital region $(\mathrm{Oz}$ according to the 10-20 system), the reference electrode was placed on the midline frontal point $(\mathrm{Fz})$ and the ground electrode on the forearm. $\mathrm{Ag} / \mathrm{AgCl}$ surface electrodes were used and their impedance was maintained below $5 \mathrm{k}$ Ohm. The responses were analyzed with a Nicolet 1000 Viking Quest, with a $1-30 \mathrm{~Hz}$ bandpass filter and a sweep time of $500 \mathrm{~ms}$. A hundred responses were averaged in each run and two runs were performed for each eye. For each subject, the latency and amplitude ("peak to peak") of the P100 component were determined for each eye, as well as relative P100 latency (interocular latency difference).

BAEP were performed with the use of stimuli presented to each ear separately via earphones. Auditory stimuli were clicks of a duration of $0.1 \mathrm{~ms}$, frequency $20.3 \mathrm{~Hz}$ and an intensity $65 \mathrm{~dB}$ higher than the hearing threshold initially established for each subject. A recording electrode was attached to the earlobe on the side of stimulation, with the reference electrode placed on the vertex $(\mathrm{Fz})$ and the ground electrode on the forearm. $\mathrm{Ag} / \mathrm{AgCl}$ surface electrodes were used and their impedance was maintained below $5 \mathrm{k} \mathrm{Ohm}$. The responses were analyzed with a Nicolet 1000 Viking Quest, with a 150-3,000 Hz bandpass filter and a sweep time of $10 \mathrm{~ms}$. Two hundred responses were averaged in each run and two runs were performed for each ear. Latencies and amplitudes ("peak to peak") were determined for components I, III and V, as well as interpeak latencies I-III, III-V, I-V and the proportion of amplitudes I/V.

\section{Statistical analysis}

Mean and median values with standard deviations were calculated for all the analyzed variables. The EP parameters obtained from the whole group of MS patients and subgroups I, II and III were compared with those from the controls, and the results were also compared between subgroups I, II and III with the use of post hoc test (Scheffe test) and then analysis of variance (ANOVA), alternatively using the Kruskal-Wallis test, when the variances in groups were not homogeneous (the homogeneity of variance was determined by the Bartelett's test) or if the number of cases was too small. Relation between continuous fatigue measures and continuous EP parameters was assessed using correlation analysis and Pearson correlation coefficients were calculated. Relation between continuous fatigue measures and categorized parameters (visual FS, brainstem FS) was assessed using correlation analysis and Spearman correlation coefficients were calculated. Multiple regression analysis was used to check the impact of age and MS-related variables upon correlations between fatigue measures and EP results. $p<0.05$ was regarded as statistically significant and $p<0.07$ sufficient to observe trends. The statistical analysis was performed using EPIINFO ver. 3.5.2 software. 


\section{Results}

On the basis of FSS/FSS-5 results, 29 patients ( 8 men, 21 women) were allocated to subgroup I (non-fatigued), 31 patients (7 men, 24 women) to subgroup II (moderately fatigued) and 26 (8 men, 18 women) to subgroup III (severely fatigued). No significant differences in terms of age or gender were found either between these groups, or between each of them and the healthy controls.

The results of MFIS on the whole group of patients ranged from 4 to 64 (mean 36.3). There was a significant correlation between MFIS results and the age of the MS patients $(R=0.24, p=0.02)$. No such correlations were found for FSS/FSS-5 results.

In MS patients the duration of the disease was 1-30 years (mean 8.57), EDSS 1-6.5 (mean 3.03) and MSSS 1.1-8.8 (mean 4.4). Visual FS scores ranged from 0 to 3 (mean 0.8), and so did Brainstem FS scores (mean 1.2). 42 patients had a history of optic neuritis, while none experienced loss of hearing during MS relapse. Patients with or without the history of optic neuritis did not differ significantly in the mean values of FSS/FSS-5 (4.63 vs $4.02, p=0.1$ ) or MFIS (39.1 vs 33.7, $p=0.12$ ). FSS/FSS5 and MFIS correlated significantly with EDSS, visual and brainstem FS (Table 1).

The mean duration of the disease was longer in subgroup III in comparison with subgroup I (11.56 vs 5.38 years, $p=0.003)$. The mean EDSS score was higher in subgroups II and III in comparison with subgroup I (2.98 vs 4.08 vs $2.12, p=0.027$ and 0.0000001 , respectively). The mean MSSS score was higher in subgroup III in comparison with subgroups II and I (5.32 vs 4.16 vs 3.86, $p=0.047$ and 0.014 , respectively).

The mean latency of the P100 component of VEP for both eyes was significantly longer in MS patients than in the controls, and so was the mean relative P100 latency (interocular latency difference). The mean P100 amplitude was significantly lower for MS patients than in controls, but only for the left eye (Table 2).

P100 latency for the right eye was significantly longer in subgroups II and III than in subgroup I, and relative P100 latency was significantly longer in subgroup III than in subgroup I (Table 2). The amplitude of P100 for the left

Table 1 Correlations between fatigue measures (FSS/FSS-5, MFIS) and degree of disability (EDSS, visual FS, brainstem FS); $R$ Spearman's correlation coefficient

\begin{tabular}{llll}
\hline & EDSS & Visual FS & Brainstem FS \\
\hline FSS/FSS-5 & $R=0.48$ & $R=0.3$ & $R=0.26$ \\
& $p=0.00001$ & $p=0.006$ & $p=0.018$ \\
MFIS & $R=0.46$ & $R=0.32$ & $R=0.24$ \\
& $p=0.0001$ & $p=0.003$ & $p=0.025$ \\
\hline
\end{tabular}

eye was lower in subgroups II and III than in the controls, and for the right eye-lower in subgroup III than in subgroup I (Table 1). No significant correlations were found between the summated values of VEP latency and amplitude and FSS/FSS-5 or MFIS. The relative latency of P100 tended to correlate positively with FSS/FSS-5 $(R=0.26$, $p=0.07)$. There was a significant correlation between summated VEP amplitude and Visual FS $(R=-0.36$, $p=0.0006)$ and a correlation on the edge of significance between summated VEP latency and Visual FS $(R=0.21$, $p=0.05)$. No correlations were found between VEP parameters and other clinical MS-related variables (duration of the disease, EDSS or MSSS).

The mean latencies of I, III and V components of BAEP on both sides did not differ significantly between MS patients and controls. Mean interlatencies I-III on the left side, III-V on the right side and I-V on both sides were significantly longer in patients than in the controls. The mean amplitude of the $\mathrm{V}$ component was significantly lower in MS patients than in the controls, but only on the right side (Table 3).

The latency of the $\mathrm{V}$ component of BAEP, and interlatency I-V for the left ear as well as interlatencies III-V and $\mathrm{I}-\mathrm{V}$ for the right ear were significantly longer in subgroup III than in the controls. In subgroup II, the latency of the V component and interlatencies III-V, and I-V for the right ear were significantly longer than in the controls (Table 3 ). The amplitude of the $\mathrm{V}$ component for the right ear was significantly lower in subgroups II and III than in the controls (Table 3). No correlations were found between the BAEP parameters (one-sided or summated values) and fatigue measures (FSS/FSS-5, MFIS). Among the BAEP parameters, interlatencies III-V and I-V showed significant positive correlations with MSSS (III-V: $R=0.28, p=0.009$; $R=0.25, p=0.024 ; \mathrm{I}-\mathrm{V}: R=0.25, p=0.021 ; R=0.2$, $p=0.05$; for left and right side, respectively). Summated latencies of BAEP components correlated significantly with Brainstem FS (I: $R=0.23, p=0.04$; III: $R=0.23$, $p=0.03$; $\mathrm{V}: R=0.36, p=0.0008)$ and so did summated amplitude of $\mathrm{V}$ component $(R=0.23, p=0.03)$. No other correlations were found between BAEP parameters and remaining clinical MS-related variables (duration of the disease, EDSS or MSSS).

\section{Discussion}

The importance of evoked potentials (EP) in the diagnosis of MS has decreased in the last decade as magnetic resonance has become the main diagnostic tool supporting clinical assessment. However, EP abnormalities are still regarded as good electrophysiological markers of disease progression and their prognostic value in the early stages of 


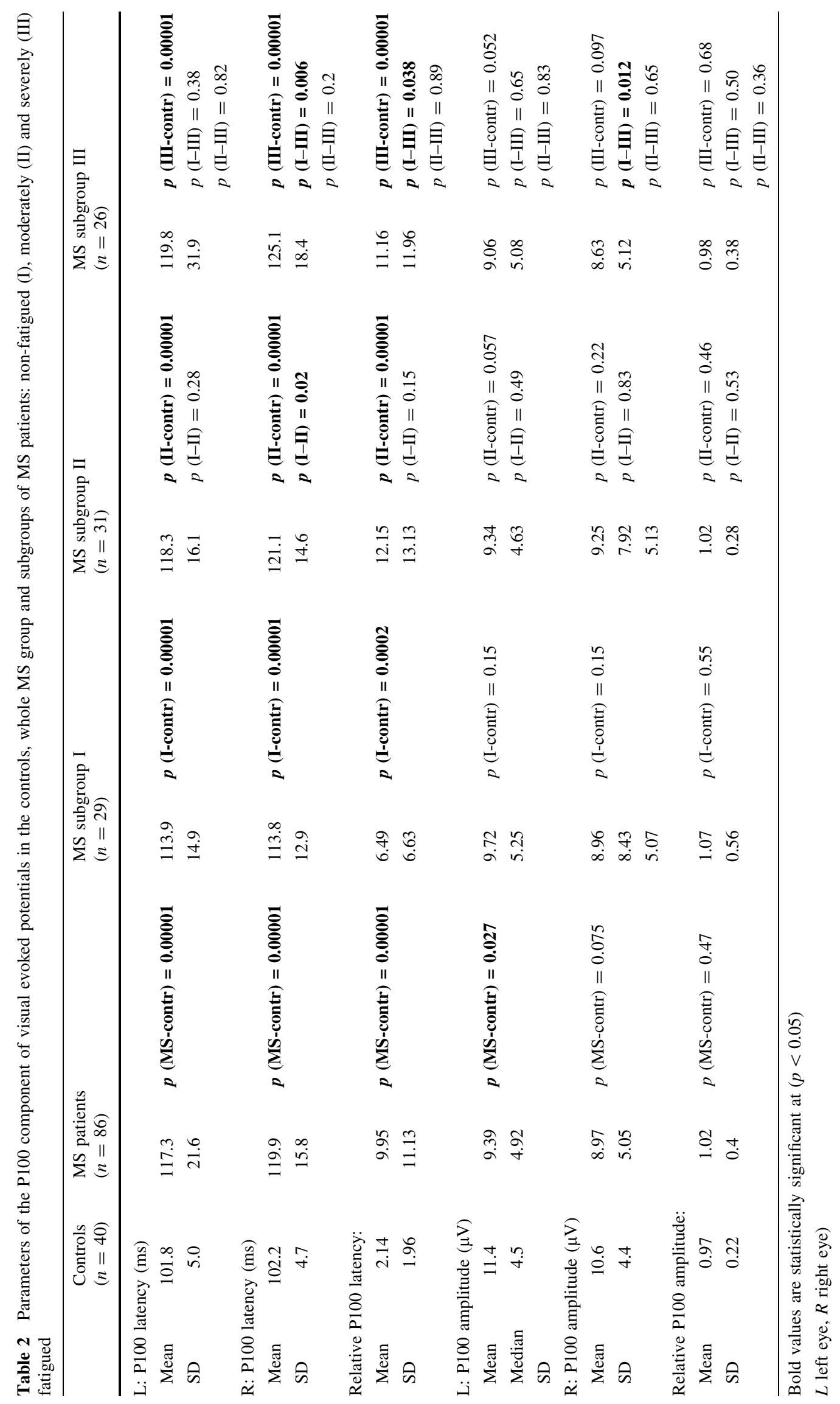




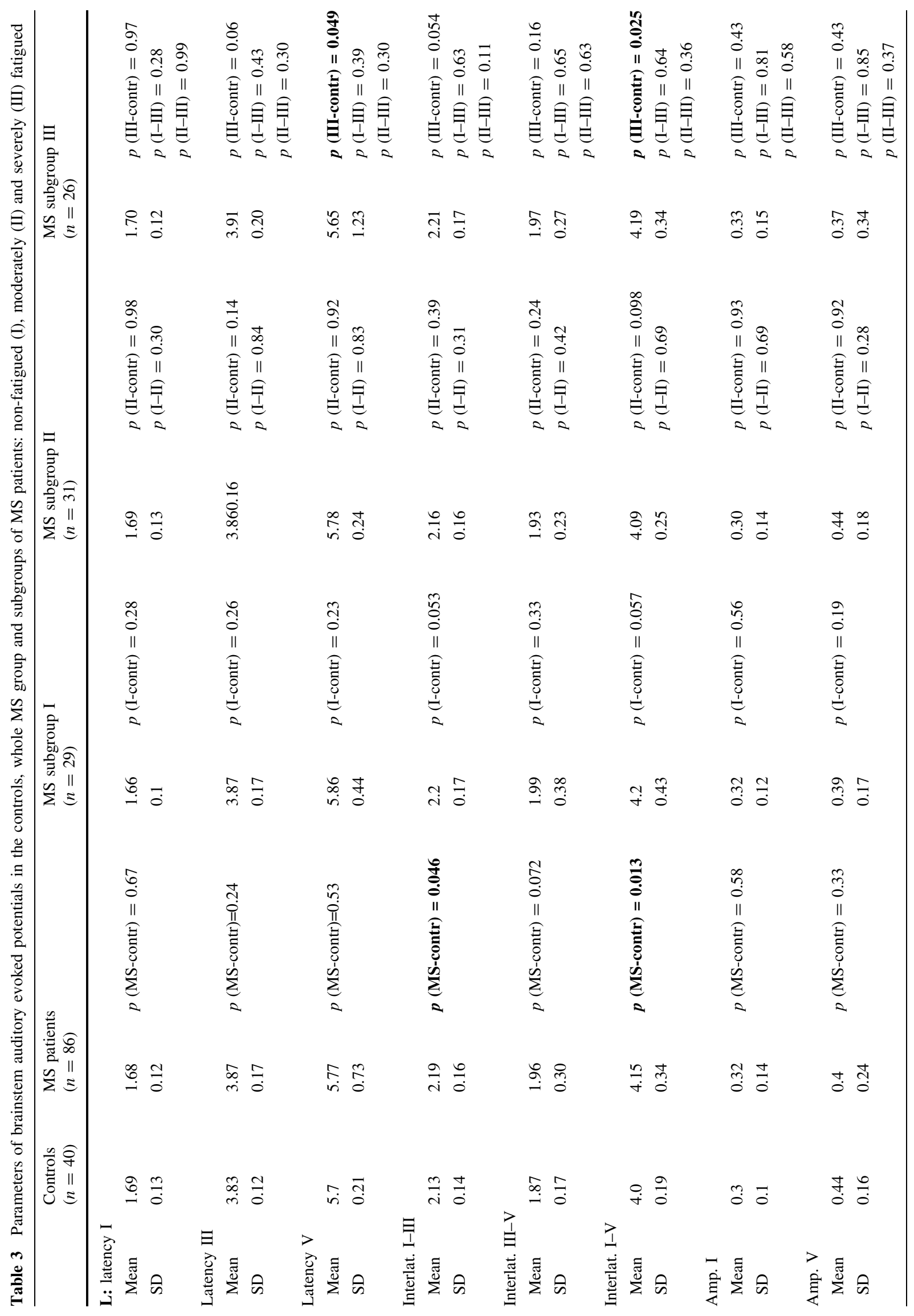




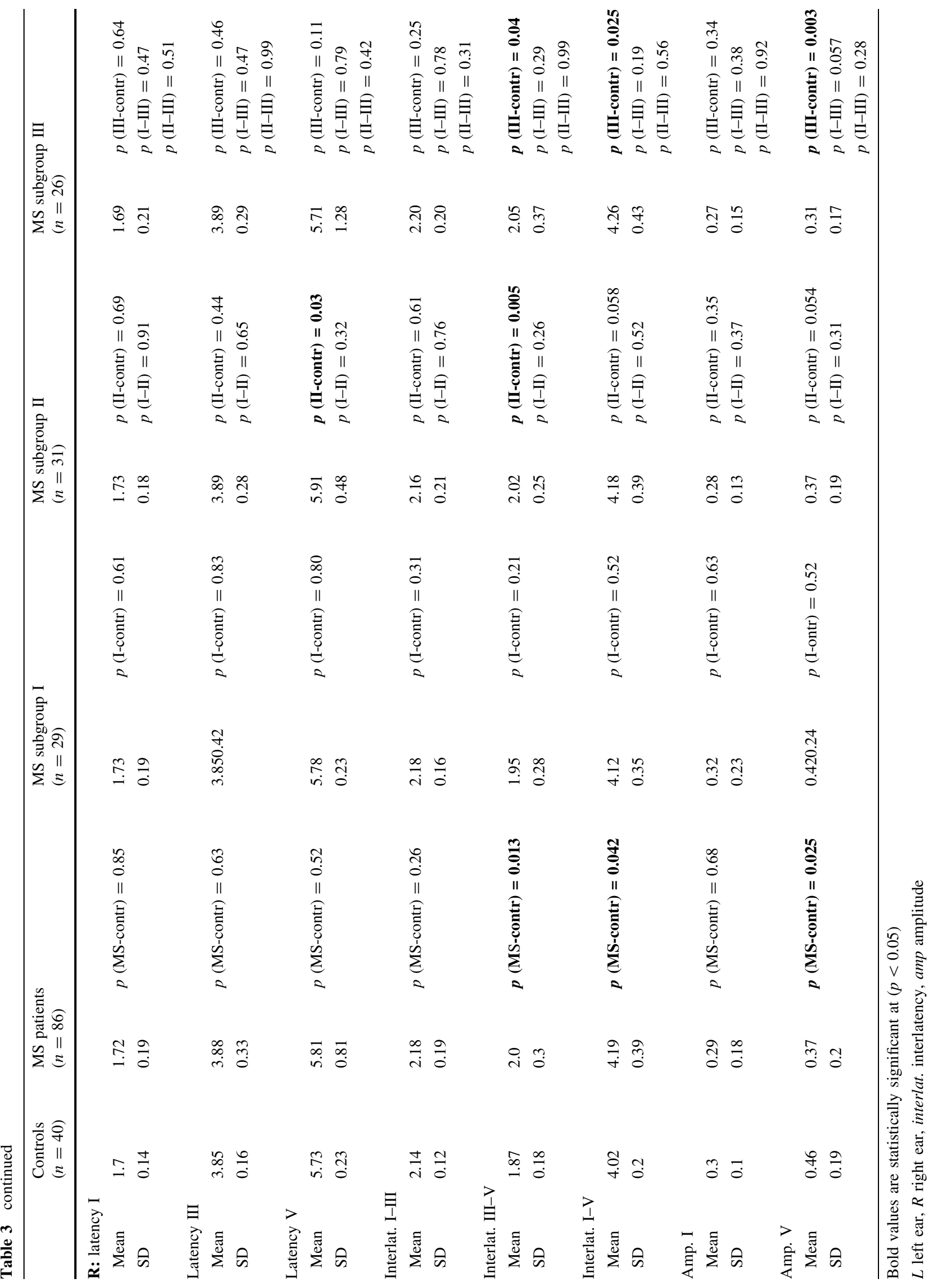


MS gains increasing attention [15-17]. Non-invasiveness and the availability of EP also encourage their use in clinical practice. Fatigue constitutes an important aspect of non-physical disability in MS patients, which is still lacking objective biomarkers, so analysis of EP parameters with regards to fatigue in MS seemed worth investigating. We deliberately chose VEP and BAEP as they have not been used in this field so far (in contrast to MEP).

Analysis of VEP showed significantly prolonged latency of the P100 component in the whole group of MS patients as well as in each of the three subgroups, when compared to the controls. Such a finding is common in MS subjects and indicates slowed conduction in the optic tract due to demyelination. It is worth noting that only severely fatigued patients in comparison with non-fatigued ones showed a significantly increased interocular latency difference (relative P100 latency). This parameter tended to correlate (although not significantly) with one of the fatigue measures (FSS/FSS-5), but-apart from visual FS score-did not show significant relationships with other disease-related variables (duration of MS, EDSS or MSSS). Although no correlation was found between relative P100 latency and the result of MFIS (which allows more detailed assessment of fatigue than FSS), it might be interesting to refer VEP results to physical and cognitive aspects of fatigue. Significant differences in P100 amplitude were also asymmetrical but they were found not only between fatigued and non-fatigued subgroups but also between MS patients and controls. The amplitude of VEP components is usually regarded as a more variable and thus less sensitive parameter than latency, so we believe P100 latency deserves more attention in further investigation.

P100 latency is known to increase with age, especially in men. Subgroups I, II and III did not differ significantly as regards age and gender structure, so the influence of demographic factors upon VEP parameters can be neglected.

In the only available study comprising MS patients (i.e., Regan et al. [18]), VEP were used to evaluate the fatigability of the visual pathway. In those patients with MS and glaucoma (but not in parkinsonic ones), the amplitude of P100 increased when additional stimuli were superimposed on the basic pattern of stimulation. Our results seem more consistent with the report of Sobieszczańska et al. [19], who assessed VEP as a measure of fatigability in healthy persons, professionally operating computer terminals. After a few hours of their constant gazing at the computer screen, there was an increase in P100 latency, a decrease in amplitude, and moreover, a decrease in correlation coefficients for the VEP parameters obtained from both hemispheres. Overall, the abnormalities of VEP parameters in our material can be attributed to the impact of MS in general, but the asymmetry of these abnormalities might have been more specific for fatigue.
Unlike the optic tract, the auditory pathway is much less frequently affected by demyelination in the course of MS. In the whole group of our MS patients in comparison with the controls, we only found significantly prolonged interlatencies between I, III and V components of BAEP, which indicate subtle conduction disturbances within the brainstem. On analysis of the subgroups of patients with and without fatigue, these abnormalities appeared to occur only in those with moderate and severe fatigue. It has to be considered that these subgroups also presented with higher level of disability and rate of its progression. The interlatencies of BAEP components indeed showed significant correlations with MSSS but not with any of the fatigue measures. Moreover, the fatigued patients also showed prolonged latency of the $\mathrm{V}$ component of BAEP (while non-fatigued ones and the whole MS group did not). This parameter, in turn, did not correlate significantly with the majority of disease-related variables, apart from Brainstem FS. It is worth noting that significant findings in BAEP parameters mostly concerned only one side.

To our knowledge, there have been no reports on BAEP with regards to fatigue in MS patients. Neri et al. [20] and Bianchedi et al. [21] described abnormalities of BAEP (the lack of component I and prolonged interlatencies) in subjects with chronic fatigue syndrome, which occurred only at higher frequencies of auditory stimulation, so were apparently revealed at a greater burden to the auditory pathway.

The relationship between fatigue and other symptoms and signs of neurological deficit remains a disputable matter [1, 2]. In our study, fatigue measures showed significant correlations with EDSS (general degree of disability, although mostly determined by ambulation skills) as well as with visual and brainstem FS scores. Fatigue is a complex phenomenon, not limited to incapability of physical effort due to motor deficit, but also possibly associated with dysfunction of other systems. Thus, VEP and BAEP, as sensitive and objective markers of visual and brainstem pathways (their parameters correlated significantly with corresponding FS scores), might provide measures of other aspects of disability contributing to fatigue.

The asymmetry of EP abnormalities in our study seemed more specifically associated with fatigue than with MS itself. Asymmetrical damage to CNS pathways interferes with the perception and integration of stimuli of particular modality. To compensate for these dysfunctions, some additional areas of the brain may become activated. This corresponds with the concept of fatigue as a result of excessive load of CNS due to dysfunction of specific areas, as is supported by neuroimaging studies involving MS patients with fatigue $[3,22,23]$.

To the best of our knowledge, so far there has been no report investigating visual and auditory EP with regards to 
fatigue in a large and well-defined group of MS patients. Abnormalities of EP in fatigued patients, independent from MS-related variables, may support the hypothesis of disturbed bioelectrical activity due to CNS damage as the background of fatigue, which contradicts the idea of its purely subjective origin. EP parameters seem promising as possible electrophysiological markers of fatigue with the asymmetry of their abnormalities deserving special attention. A limitation of our study is the fact that the assessment of fatigue and EP parameters was performed only once, without re-testing to check for reliability of the results. Considering the common fluctuations of MS symptoms and the variability of EP parameters, we have already planned further study including parallel monitoring of fatigue and EP in the course of the disease to evaluate their relationships in prospective observation.

In conclusion, the parameters of VEP and BAEP undergo significant, mostly asymmetrical changes in MS patients with moderate and severe fatigue. These findings seem to support the hypothesis of neuronal pathways dysfunction as the background of MS fatigue. The role of EP parameters as electrophysiological markers of fatigue seems promising and deserves further investigation.

Conflict of interest The authors declare that they have no conflict of interest.

Open Access This article is distributed under the terms of the Creative Commons Attribution License which permits any use, distribution, and reproduction in any medium, provided the original author(s) and the source are credited.

\section{References}

1. Comi G, Leocani L, Rossi P, Colombo B (2001) Physiopathology and treatment of fatigue in multiple sclerosis. J Neurol 248:174-179

2. Kos D, Kerckhofs E, Nagels G, D'hooghe MB, Ilsbroukx S (2008) Origin of fatigue in multiple sclerosis: review of the literature. Neurorehabil Neural Repair 22:91-102

3. Leocani L, Colombo B, Comi G (2008) Physiopathology of fatigue in multiple sclerosis. Neurol Sci 29(Suppl 2):S241-S243

4. Vucic S, Burke D, Kiernan MC (2010) Fatigue in multiple sclerosis: mechanisms and management. Clin Neurophysiol 121:809-817

5. Colombo B, Boneschi FM, Rossi P, Rovaris M, Maderna L, Filippi M, Comi G (2000) MRI and motor evoked potential findings in nondisabled multiple sclerosis patients with and without symptoms of fatigue. J Neurol 247:506-509

6. Sheean GL, Murray NM, Rothwell JC, Miller DH, Thompson AJ (1997) An electrophysiological study of the mechanism of fatigue in multiple sclerosis. Brain 120(Suppl 2):299-315

7. Liepert J, Mingers D, Heesen C, Baumer T, Weiller C (2005) Motor cortex excitability and fatigue in multiple sclerosis: a transcranial magnetic stimulation study. Mult Scler 11:316-321

8. Gandevia SC, Allen GM, Butler GE, Taylor JL (1996) Supraspinal factors in human muscle fatigue: evidence for suboptimal output from the motor cortex. J Physiol 490:529-536
9. Polman CH, Reingold SC, Banwell B, Clanet M, Cohen JA, Filippi M, Fujihara K, Havrdova E, Hutchinson M, Kappos L, Lublin FD, Montalban X, O'Connor P, Sandberg-Wollheim M, Thompson AJ, Waubant E, Weinshenker B, Wolinsky JS (2011) Diagnostic criteria for multiple sclerosis: 2010 revisions to the McDonald criteria. Ann Neurol 69:292-302

10. Kurtzke JM (1983) Rating neurological impairment in multiple sclerosis: an expanded disability status scale. Neurology 33:1444-1452

11. Roxburgh RHSR, Seaman SR, Masterman T, Hensiek AE, Sawcer SJ, Vukusic S, Achiti I, Confavreux C, Coustans M, le Page E, Edan G, McDonnell GV, Hawkins S, Trojano M, Liguori M, Cocco E, Marrosu MG, Tesser F, Leone MA, Weber A, Zipp F, Miterski B, Epplen JT, Oturai A, Sørensen PS, Celius EG, Lara NT, Montalban X, Villoslada P, Silva AM, Marta M, Leite I, Dubois B, Rubio J, Butzkueven H, Kilpatrick T, Mycko MP, Selmaj KW, Rio ME, Sá M, Salemi G, Savettieri G, Hillert J, Compston DA (2005) Multiple sclerosis severity score. Using disability and disease duration to rate disease severity. Neurology 64:1144-1151

12. Krupp LB, LaRocca NG, Muir-Nash J, Steinberg AD (1989) The fatigue severity scale. Application to patients with multiple sclerosis and systemic lupus erythematosus. Arch Neurol 46:1121-1123

13. (1998) Fatigue and multiple sclerosis: evidence-based management strategies for fatigue in multiple sclerosis. Multiple Sclerosis Council for Clinical practice guidelines

14. Mills RJ, Young CA, Nicholas RS, Pallant JF, Tennant A (2009) Rasch analysis of the Fatigue Severity Scale in multiple sclerosis. Mult Scler 15:81-88

15. Leocani L, Comi G (2008) Neurophysiological markers. Neurol Sci 29(Suppl 2):S218-S221

16. Margaritella N, Mendozzi L, Tronci F, Colicino E, Garegnani M, Nemni R, Gilardi E, Pugnetti L (2013) The evoked potentials score improves the identification of benign MS without cognitive impairment. Eur J Neurol 20:1423-1425

17. Ramanathan S, Lenton K, Burke T, Gomes L, Storchenegger K, Yiannikas C, Vucic S (2013) The utility of multimodal evoked potentials in multiple sclerosis prognostication. J Clin Neurosci 20:1576-1581

18. Regan D, Neima D (1984) Visual fatigue and visual evoked potentials in multiple sclerosis, glaucoma, ocular hypertension and Parkinson's disease. J Neurol Neurosurg Psychiatry 47:673-678

19. Sobieszczańska M, Salomon E, Borodulin-Nadzieja L, Pilecki W, Jagielski J, Kałka-Gebala R, Janocha A, Kałka D (1998) The evaluation of central visual fatigue in computer terminal users by visual evoked potentials. Neurol Neurochir Pol 32:1369-1375

20. Neri G, Bianchedi M, Croce A, Moretti A (1996) "Prolonged" decay test and auditory brainstem responses in the clinical diagnosis of the chronic fatigue syndrome. Acta Otorhinolaryngol Ital 16:317-323

21. Bianchedi M, Croce A, Moretti A, Neri G, Barberio A, Iezzi A, Pizzigallo E (1995) Auditory brain stem evoked potentials in the evaluation of chronic fatigue syndrome. Acta Otorhinolaryngol Ital 15:403-410

22. Roelcke U, Kappos L, Lechner-Scott J, Brunnschweiler H, Huber S, Ammann W, Plohmann A, Dellas S, Maguire RP, Missimer J, Radü EW, Steck A, Leenders KL (1997) Reduced glucose metabolism in the frontal cortex and basal ganglia of multiple sclerosis patients with fatigue: a F-18-fluorodeoxyglucose positron emission tomography study. Neurology 48:1566-1571

23. Filippi M, Rocca MA, Colombo B, Falini A, Codella M, Scotti G, Comi G (2002) Functional magnetic resonance imaging correlates of fatigue in multiple sclerosis. Neuroimage 15:559-567 